\title{
LETTER \\ DWT Domain On-Line Signature Verification Using the Pen-Movement Vector
}

\author{
Isao NAKANISHI ${ }^{\dagger a}$, Member, Hiroyuki SAKAMOTO ${ }^{\dagger \dagger}$, Student Member, Naoto NISHIGUCHI ${ }^{\dagger \dagger *}$, \\ Yoshio ITOH ${ }^{\dagger \dagger}$, Members, and Yutaka FUKUI ${ }^{\dagger \dagger}$, Fellow
}

\begin{abstract}
SUMMARY In order to reduce the computational complexity of the DWT domain on-line signature verification, the authors propose to utilize the pen-movement vector as an input parameter. Experimental results indicate that the verification rate obtained using the pen-movement vector parameter is equivalent to that obtained by the conventional method, although the computational complexity of the proposed method is approximately half that of the conventional method.
\end{abstract}

key words: on-line signature verification, DWT, pen-movement vector, computational complexity

\section{Introduction}

As information services over the Internet such as Electronic Commerce (EC) and electronic data interchange come to be widely used, biometrics for user authentication has attracted a great deal of attention [1]. The authors have proposed an on-line signature verification method based on Discrete Wavelet Transform (DWT) and adaptive signal processing [2]-[4]. However, the computational complexity** of the conventional method is high because $\mathrm{x}$ and $\mathrm{y}$ coordinate data are separately processed as a pen-position parameter.

The present paper proposes the adoption of the penmovement vector as an on-line signature parameter. The pen-movement vector is derived from $\mathrm{x}$ and y coordinates, so that the computational complexity is approximately half that of the conventional method. As well as the conventional method [2]-[4], the time-varying signal of the penmovement vector is decomposed into sub-band signals by DWT and then verification is achieved by adaptive signal processing in each sub-band. The overall decision for verification is made by combining the verification results obtained for the sub-bands. Verification experiments are carried out to confirm the performance of the proposed DWT domain on-line signature verification using the penmovement vector.

\section{Pen-Movement Vector}

The pen-position parameter consists of discrete time-

Manuscript received November 28, 2005.

Final manuscript received December 19, 2005.

${ }^{\dagger}$ The author is with the Faculty of Regional Sciences, Tottori University, Tottori-shi, 680-8552 Japan.

${ }^{\dagger}$ The author is with the Faculty of Engineering, Tottori University, Tottori-shi, 680-8552 Japan.

${ }^{*}$ Presently, with the Mitsubishi Electric Corporation.

a)E-mail: isao@rstu.jp

DOI: 10.1093/ietfec/e89-a.4.1129 varying signals of $\mathrm{x}$ and $\mathrm{y}$ coordinates, which are presented as $x^{*}\left(n^{\prime}\right)$ and $y^{*}\left(n^{\prime}\right)$, where $n^{\prime}\left(=0,1, \cdots, N_{\max }-1\right)$ is a sampled time index and $N_{\max }$ is the number of sampled data. Moreover, the pen-position parameter is generally normalized to reduce intra-class variation in the signatures of individuals.

$$
\begin{aligned}
& x(n)=\frac{x^{*}(n)-x_{\text {min }}}{x_{\text {max }}-x_{\text {min }}} \cdot \alpha_{x} \\
& y(n)=\frac{y^{*}(n)-y_{\text {min }}}{y_{\max }-y_{\min }} \cdot \alpha_{y}
\end{aligned}
$$

where $n(=0 \sim 1)$ is the normalized sampled time index given by $n=n^{\prime} /\left(N_{\max }-1\right), x_{\max }$ and $y_{\max }$ are the maximum and minimum values of $x^{*}(n)$ and $y^{*}(n)$, respectively, and $\alpha_{x}$ and $\alpha_{y}$ are scaling factors for avoiding underflow calculation in sub-band decomposition. These $x(n)$ and $y(n)$ are separately processed in the conventional method [2]-[4], so that the computational complexity is high.

Next, the pen-movement vector parameter $v(n)$ is defined as follows:

$$
v(n)=d(n) \cdot \theta(n)
$$

where $d(n)$ and $\theta(n)$ are the pen-movement distance and penmovement angle, respectively. The pen-movement distance and pen-movement angle are derived from the pen-position, as shown in Fig. 1, and are formulated as follows:

$$
d(n)=\sqrt{\Delta x(n)^{2}+\Delta y(n)^{2}} / s
$$

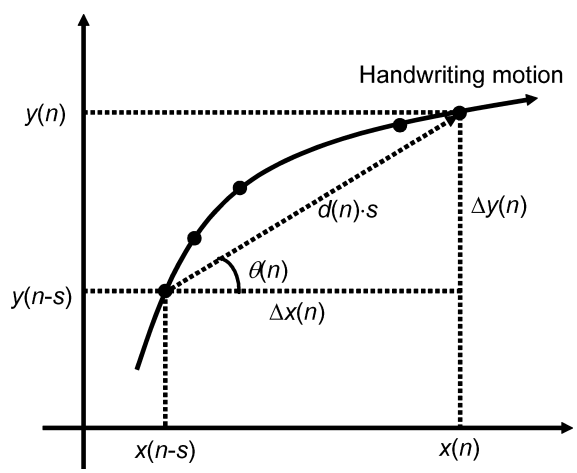

Fig. 1 Definitions of pen-movement distance $d(n)$ and pen-movement angle $\theta(n)$.

${ }^{* *}$ There are two dimensions of computational complexity, time and space. In this paper, the time complexity is examined as computational complexity. 


$$
\theta(n)= \begin{cases}\tan ^{-1} \frac{\Delta y(n)}{\Delta x(n)}, & \Delta x(n)>0 \\ \tan ^{-1} \operatorname{sgn}(\Delta y(n)) \cdot \frac{\pi}{2}, & \Delta x(n)=0 \\ \tan ^{-1} \frac{\Delta y(n)}{\Delta x(n)}+\pi, & \Delta x(n)<0, \Delta y(n) \geq 0 \\ \tan ^{-1} \frac{\Delta y(n)}{\Delta x(n)}-\pi, & \Delta x(n)<0, \Delta y(n)<0\end{cases}
$$

where

$$
\begin{aligned}
& \Delta x(n)=x(n)-x(n-s) \\
& \Delta y(n)=y(n)-y(n-s)
\end{aligned}
$$

and $s$ represents the time shift.

In this way, only one parameter is processed in the proposed method, so that the computational complexity becomes approximately half that of the conventional method which processes $x(n)$ and $y(n)$ separately. The details are examined in the next section.

In the following, it is explained why the pen-movement vector is adopted. The pen-movement distance $d(n)$ and pen-movement angle $\theta(n)$ are easily obtained by combining $\mathrm{x}$ and $\mathrm{y}$ coordinate data. However, $d(n)$ is a parameter of length and does not have two-dimensional characteristics. On the other hand, $\theta(n)$ has two-dimensional characteristics but it lacks for the information on distance. Actually, it was found that the verification performance using $d(n)$ or $\theta(n)$ was slightly degraded comparing with the conventional method. Resultingly the authors propose the pen-movement vector $v(n)$ by multiplying $d(n)$ and $\theta(n)$.

It is pointed out that the verification system using the pen-movement vector can not distinguish $v(n)=d(n) / 2$. $2 \theta(n)$ from $v(n)=d(n) \cdot \theta(n)$. However, it is rare that all penmovement vector data of a signature fall into such indistinguishable condition. Even if such a rare case is happened, it is possible to detect and reject the same pen-movement vector data in the enrollment phase. In the proposed verification system, genuine signature data are enrolled as the template in advance. In addition, the signature has an advantage that it is changeable. Therefore, the problem of indistinguishable pen-movement vector can be avoided by the total system.

The procedure for the on-line signature verification using the pen-movement vector is similar to that for the conventional method [2]-[4]; therefore, its explanation is omitted here for brevity. Please refer to Refs. [2]-[4] for details.

\section{Computational Complexity}

The proposed method consists of not only a sub-band filter bank and adaptive signal processing but also procedures for making a template, the decision of the decomposition level and stroke matching, as described in Sects. 4.3-4.5 of Ref. [3]. Therefore, software processing using the digital signal processor (DSP) is considered to be suitable for implementation.

Figure 2 shows a comparison of computational complexity, in which (a) is a block diagram of the conventional method [2]-[4] and (b) is a block diagram of the proposed method, where SD, VR, OD and PMV correspond to sub-

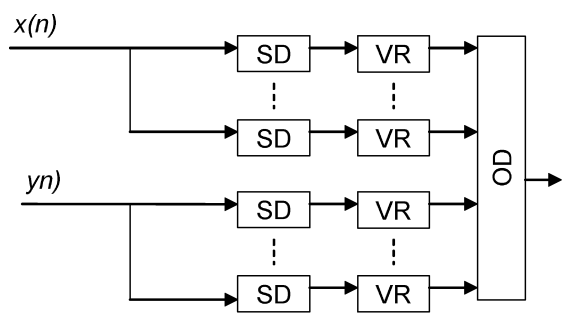

(a)

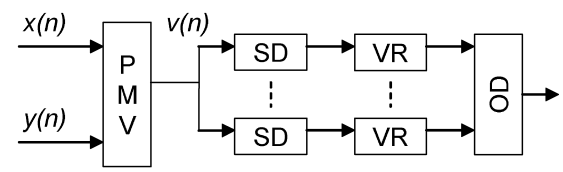

(b)

Fig. 2 Comparison of computational complexity.

band decomposition, verification using adaptive signal processing, overall decision and pen-movement vector blocks, respectively. In the following, the primary topic of discussion is the number of multiplications and divisions. The sub-band decomposition consists of analysis and synthesis filters. Therefore, its calculation is based on multiplication and accumulation. Verification by adaptive signal processing is based on multiplication and division, as defined by Eqs. (18)-(22) in Ref. [3]. Moreover, these calculations are achieved in parallel at all sub-bands. Concretely, $(2 \times F L+6) \times M d$ multiplications and $6 \times M d$ divisions are required, where $F L$ is the filter length [3]. These multiplications and divisions can be reduced by introducing the pen-movement vector.

On the other hand, the pen-movement vector newly requires the calculation of Eqs. (4)-(7). In order to accelerate the calculation of Eq. (5), Look-Up Table (LUT) can be used while it increases memory. Thus, three multiplications, two divisions and one root operation are required. In general, root operations and divisions require large calculation times. Assuming that the calculation time of the root operation is equivalent to that of division, three multiplications and three divisions are required by the introduction of the pen-movement vector. Based on these comparisons, it is confirmed that the introduction of the pen-movement vector is effective for reducing the computational complexity of the DWT domain on-line signature verification and then the computational complexity of the proposed method is considered to be approximately half that of the conventional method.

\section{Experimental Results}

In order to verify the performance of the proposed on-line signature verification using the pen-movement vector, a verification experiment was performed. The conditions of the experiment were identical to those described in Sect. 5 of [3]. The processing parameters are as follows:

- Scaling parameter: $\alpha_{x}=\alpha_{y}=100$

- Time shift in pen-movement vector: $s=8$ 


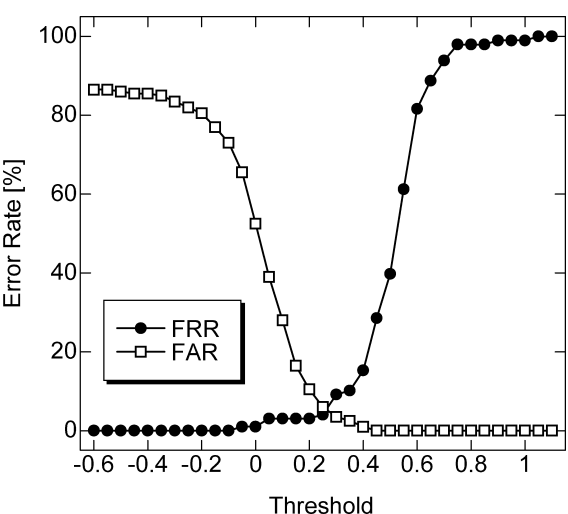

Fig. 3 Verification results.

- Wavelet function: Daubechies8

- Maximum decomposition level: $M d_{\max }=8$

- Number of genuine signatures for template: $T=5$

- Number of processed levels: $L=4$

In the previously proposed methods, Daubechies8 has been confirmed to be effective as the wavelet function [2]-[4]. Therefore, Daubechies8 is adopted in the present study, since the purpose of the present study is to reduce computational complexity of the DWT domain on-line signature verification method while maintaining its performance.

Figure 3 shows the False Rejection Rate (FRR) and False Acceptance Rate (FAR) with respect to the threshold value. In general, verification performance is estimated by the Equal Error Rate (EER), where the FRR and the FAR are the same. The EER was approximately $5 \%$ when the threshold value was approximately 0.25 . This rate is equivalent to that of the conventional method [2]-[4], whereas the computational complexity is reduced to half that of the conventional method.

\section{Conclusion}

DWT domain on-line signature verification using the pen- movement vector was proposed. The pen-movement vector was easily derived from $\mathrm{x}$ and $\mathrm{y}$ coordinates, which were used in the conventional method. Therefore, the computational complexity was reduced by half compared to the conventional method. Verification experiments revealed that the verification rate was approximately $95 \%$, which was equivalent to that of the conventional method. Thus, for the proposed method, a verification rate equivalent to that of the conventional method was achieved at half of its computational complexity. It must be studied further to implement the proposed verifiaction system on the DSP.

\section{Acknowledgments}

This research was supported in part by the Electric Technology Research Foundation of Chugoku and the Grant-inAid for Scientific Research (No.17560340) from the Ministry of Education, Culture, Sports, Science and Technology of Japan.

\section{References}

[1] A. Jain, R. Bolle, and S. Pankanti, BIOMETRICS Personal Identification in Networked Society, Kluwer Academic Publishers, Massachusetts, 1999.

[2] I. Nakanishi, N. Nishiguchi, Y. Itoh, and Y. Fukui, "On-line signature verification method utilizing feature extraction based on DWT,' Proc. 2003 IEEE International Symposium on Circuits and Systems (ISCAS2003), vol.IV, pp.73-76, Bangkok, Thailand, May 2003.

[3] I. Nakanishi, N. Nishiguchi, Y. Itoh, and Y. Fukui, "On-line signature verification based on subband decomposition by DWT and adaptive signal processing," IEICE Trans. Fundamentals (Japanese Edition), vol.J87-A, no.6, pp.805-815, June 2004.

English translation is published in Electronics and Communications in Japan, Part 3, Scripta Technica, 2005.

[4] I. Nakanishi, N. Nishiguchi, Y. Itoh, and Y. Fukui, "On-line signature verification based on discrete wavelet domain adaptive signal processing," Proc. First International Conference on Biometric Authentication (ICBA2004), pp.584-591, Hong Kong, China, July 2004. 